\title{
Safety and efficacy of paediatric silicone Ahmed glaucoma valve (AGV) in adult eyes with post-VR surgery glaucoma
}

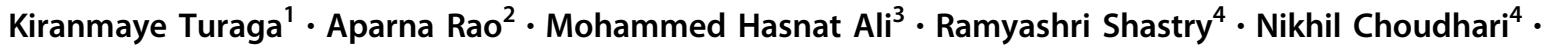 \\ Chandrasekhar Garudadri ${ }^{4} \cdot$ Sirisha Senthil ${ }^{4}$
}

Received: 11 June 2019 / Revised: 6 August 2019 / Accepted: 2 September 2019 / Published online: 4 November 2019

(c) The Author(s), under exclusive licence to The Royal College of Ophthalmologists 2019

\begin{abstract}
Purpose To compare the safety and efficacy of the FP8 AGV (paediatric) with the standard FP7 AGV in adult postvitreoretinal (VR) surgery glaucoma.

Methods We included 45 consecutive eyes with post-VR surgery glaucoma implanted with either FP8 $(n=28)$ or FP7 $(n=$ 17) AGV between 2008 and 2016. The data analysed included visual acuity (VA), intraocular pressure (IOP), complications, interventions, and outcomes.

Results Mean age $(p=0.24)$, mean baseline VA $(p=0.77)$, preoperative IOP $(p=0.41)$, number of antiglaucoma medications ( $p=0.92)$, and previous surgeries $(p=0.16)$ were comparable in the two groups. The number of eyes with previous belt buckle was significantly higher $(p<0.001)$ in the FP8 group $(17 / 28)$ compared with the FP7 group $(2 / 17)$, indicating space constraint and scarred conjunctiva. The median follow-up (25th, 75th percentile) was $14.7(9.1,25.3)$ months in the FP7 and $9.8(6.4,34.7)$ months in the FP8 groups $(p=0.62)$. Postoperatively, the median VA $(p=0.24)$, the mean IOP at final follow-up $(p=0.15)$, and median number of AGM $(p=0.99)$ were comparable in both the groups. The median percentage drop in IOP (95\% confidence interval) with the FP7 implant was 55\% (43.70\%) and with FP8 implant was 53.8\% $(25,73),(p=0.20)$. None in the FP7 group failed, while three eyes in the FP8 group failed. During the study period, two eyes in the FP8 group had tube exposure that was surgically managed. None of the eyes had implant exposure or loss of light perception.

Conclusion Use of the paediatric FP8 AGV in adult post-VR surgery glaucoma eyes with severe conjunctival scarring and limited subconjunctival space resulted in reasonable IOP control compared with the standard FP7 AGV implant. There were no eyes with implant extrusion.
\end{abstract}

\section{Introduction}

Managing glaucoma post-vitreoretinal (VR) surgery is challenging. Glaucoma drainage devices offer better longterm intraocular pressure (IOP) control in these eyes

Sirisha Senthil

sirishasenthil@lvpei.org

1 L.V. Prasad Eye Institute, GMR Varalakshmi Campus, Visakhapatnam, India

2 L.V. Prasad Eye Institute, MTC Campus, Bhubaneswar, India

3 Clinical Epidemiology and Bio-Statistics, L.V. Prasad Eye Institute, Hyderabad, India

4 VST Glaucoma Center, L.V. Prasad Eye Institute, Hyderabad, India compared with trabeculectomy or cyclodestructive procedures [1-7]. Severely scarred conjunctiva with encircling band/buckle makes the implantation of a large drainage device challenging. In addition, the conjunctival closure is difficult, predisposing these eyes to implant exposure and extrusion [8]. There are very few reports on the outcomes and complications of Ahmed glaucoma valve (AGV) implanted in eyes with post-VR surgery glaucoma. Gupta et al. reported moderate success with IOP control and high rate of complications following FP7 implantation in eyes with post-VR surgery glaucoma [8]. Nearly half the eyes in their series (48\%) had complications, and about a quarter (22\%) had sight threatening complications [8]. Inadequate dissection, larger implant size, and improperly placed implant are factors that could contribute to implant exposure or extrusion in eyes with scarred conjunctiva. There are no studies reporting the outcomes with smaller AGV (FP8) in 
these eyes. However, in post-VR surgery eyes with severe conjunctival scarring or encircling buckle/band, there are reports of amputated or trimmed FP7 or Baerveldt implants placed in the restricted subconjunctival space $[9,10]$.

The AGV (New World Medical Inc., Rancho Cucamonga, CA, USA), a pseudo valve, is a flow-restrictive aqueous drainage implant available in two models. The FP7 model is $13 \mathrm{~mm}$ wide and $16 \mathrm{~mm}$ long with a surface area of $184 \mathrm{~mm}^{2}$, while the FP8 model is $11 \mathrm{~mm}$ wide and $11 \mathrm{~mm}$ long with a surface area of $102 \mathrm{~mm}^{2}$. It is technically challenging to place a larger FP7 implant in eyes with shorter axial lengths; excessive conjunctival scarring due to previous intraocular/extraocular surgeries; and eyes with encircling bands or buckle. The FP8 implants are not preferred for use in adult eyes, due to the smaller surface area and possibility of lesser IOP control. However, these implants may be beneficial in situations with limited subconjunctival space. The only report that compared the outcomes of the FP7 and FP8 in adult eyes with refractory glaucoma was published by Koh et al. [11]. They reported similar success rates and complications with the two models at 3-year follow-up. However, they excluded eyes with severe conjunctival scarring, and eyes post retinal detachment surgery with scleral buckle or vitrectomy, in their study [11]. Intuitively, implantation of the FP8 model of AGV with a smaller surface area would be easier and probably safer in post VR surgery eyes with restricted subconjunctival space compared with FP7. However, there are no reports comparing the two models in post-VR surgery eyes to date. Hence, we chose to study the safety and efficacy of AGV implantation using these two models (FP7 and FP8) in adult eyes with post-VR surgery refractory glaucoma.

\section{Methods}

We analysed the medical records of patients who underwent AGV implantation for control of IOP post-VR surgery glaucoma at L.V. Prasad Eye Institute, in a retrospective study. The study was approved by the institutional ethics committee, and informed consent for surgery was obtained. we also obtained a general consent for using anonymized clinical data for research from all participants, as per the institute's protocol. All methods adhered to the declaration of Helsinki for research involving human subjects. The inclusion criteria for patients was age $>18$ years, treated by two glaucoma specialists between September 20016 and October 2016 for secondary glaucoma post-VR surgery, and who underwent AGV implantation by one of the two specialists for medically uncontrolled glaucoma. We included one eye per patient. The exclusion criteria comprised those who underwent combined retinal and glaucoma surgery, subjects $<18$ years, and postoperative follow-up less than 3 months. Our protocol for management of secondary glaucoma post-VR surgery was as follows: early or late postoperative rise in IOP was managed with medical treatment including oral carbonic anhydrase inhibitors. Surgical intervention was planned in cases with persistent elevation in IOP despite maximum topical medications $(>1$ visit) and/or need for prolonged oral carbonic anhydrase inhibitor for IOP control, intolerance to medications, and in the presence of disc and field progression. The parameters recorded were indication for VR surgery, number and type of VR surgeries, number of antiglaucoma medications (AGM), visual acuity (VA), IOP with Goldmann applanation tonometer, anterior segment details, fundus examination with a 78 dioptre lens, and whenever possible 24-2 Swedish Interactive Threshold Algorithm standard automated visual field test (Humphrey visual field analyzer; Carl Zeiss Meditec, Dublin, CA, USA). The staff optometrist performed the IOP measurements. The model of AGV, implant location, presence of encircling band, and any intraoperative complications were recorded.

The follow up visits were scheduled at 1 day, 1 week, and 1 month postoperatively and every 3-6 months thereafter. Detailed data were recorded during each follow up visit. The patients' local ophthalmologists were contacted to update the records, for those patients who preferred to follow up with them locally. Surgical success was defined as IOP $>5$ and $\leq 21 \mathrm{mmHg}$ (with or without topical AGM). Failure was defined as IOP $\leq 5$ or $>21 \mathrm{mmHg}$, on two consecutive follow-up visits after 3 months, repeat intervention for IOP control, or a sight threatening complication.

\section{Surgical procedure}

The procedure involved exposing surgical site with a corneal traction suture, and performing conjunctival peritomy 3-4 $\mathrm{mm}$ from the limbus, creating a wide subconjunctival pocket posteriorly between the two recti muscles. Care was taken to perform gentle dissection on either side of the conjunctival pocket, followed by gentle bipolar cautery. The implant was primed gently by injecting $1-2 \mathrm{ml}$ balanced salt solution. The plate of the implant was secured to the sclera, $8-10 \mathrm{~mm}$ posterior to the limbus, with $10-0$ or 9-0 polypropylene sutures (Ethilon; Ethicon Inc., Somerville, NJ). In the presence of encircling band, the implant plate was secured to the sclera posterior to the buckle, or over the buckle when space did not permit, and secured to the silicone band. The tube was trimmed to a desired length with the bevel facing up, and was inserted into the anterior chamber or sulcus through a $3 \mathrm{~mm}$ track created using a 24gauge needle. The tube was anchored to the episcleral tissue with one 10-0 nylon mattress suture. A rectangular donor 
scleral patch graft was used to cover the entire subconjunctival tube and fixed with fibrin glue (Baxter Healthcare, One Baxter Parkway, Deerfield, IL) and additional 10-0 nylon sutures. The conjunctival incision was closed using 8-0 polyglactin suture in a continuous fashion. The postoperative treatment included moxifloxacin $0.5 \%$ eye drops four times per day for 1 week, tapering doses of prednisolone acetate $1 \%$ eye drops over 4-6 weeks, and topical cycloplegic homatropine hydrobromide $2 \%$ eye drops twice (2-3 times) a day for 2-3 weeks.

\section{Statistical analyses}

Shapiro-Wilk test was used to assess the Gaussian distribution of the study population. Descriptive statistics are represented as mean \pm standard deviation or median and interquartile range based on their distribution. Independent Student's $t$ test and chi-squared tests were performed to compare ocular and demographic variables between the two groups. Kaplan-Meier analysis was performed to estimate the success rate over time. Ranksum test was performed to compare the success rate of surgery, percentage reduction in IOP, and reduction in AGM between the two groups. A $p$ value of $<0.05$ was considered statistically significant. Statistical analyses were performed using 'R' software.

\section{Results}

We included 58 eyes of 58 subjects who underwent AGV implantation in eyes with post-VR surgery refractory glaucoma between September 2008 and October 2016. Among these, 13 eyes of 13 subjects with follow up less than 3 months were excluded; hence only 45 eyes were included for the analysis. Of the 45 eyes, 17 eyes underwent FP7 implantation and 28 eyes underwent FP8 implantation. Table 1 summarizes the demographic and clinical characteristics of the study population. The mean age of the FP7 and FP8 groups was $43.7 \pm 18.7$ years and $37.8 \pm 15.7$ years, respectively, $(p=0.24)$. Preoperative IOP was $32.9 \pm 7.8 \mathrm{mmHg}$ in the FP7 group and $30.6 \pm 10.2 \mathrm{mmHg}$ in the FP8 group $(p=0.41)$. The mean cup-to-disc ratio in both the groups was 0.9 indicating severe glaucoma. The two groups were comparable with regard to baseline VA $(p=0.7)$, number of preoperative AGM $(p=0.92)$, previous glaucoma surgeries $(p=0.8)$, and site of implant placement $(p=0.4)$.

The number of eyes with previous belt buckle was significantly higher in the FP8 group compared with the FP7 group (2/17 in FP7 group and 17/28 in FP8 group, $p<$ 0.001). One third of the eyes in both the groups had failed glaucoma surgeries in the past. Mean number of nonglaucoma intraocular surgeries was $3.3 \pm 1.4$ in the FP8 group and $2.7 \pm 1.1$ in the FP7 group, $(p=0.16)$.

Table 1 Comparison of clinical characteristics between the FP7 and FP8 groups

\begin{tabular}{llll}
\hline Parameters & FP7 group $(n=17)$ & FP8 group $(n=28)$ & $p$ value \\
\hline Mean age years (SD) & $43.7 \pm 18.7$ & $37.8 \pm 15.9$ & $0.25^{\mathrm{a}}$ \\
Sex (F:M) & $8: 7$ & $7: 21$ & $0.36^{\mathrm{b}}$ \\
Median baseline BCVA (Log MAR) & $0.9(0.6,1)$ & $4(0.6,1.3)$ & $0.77^{\mathrm{c}}$ \\
Median number of preoperative AGM & $4(3,4)$ & $30.6 \pm 10.2$ & $0.92^{\mathrm{c}}$ \\
Mean preoperative IOP (mmHg) & $32 \pm 7.8$ & $0.9(0.8,0.9)$ & $3.3 \pm 1.4$ \\
Median cup-to-disc ratio & $0.9(0.8,0.9)$ & $1(0.7,1.4)$ \\
Mean number of previous intraocular surgeries & $2.7 \pm 1.1$ & $0(0,1)$ & $0.41^{\mathrm{a}}$ \\
Median Log MAR visual acuity at final follow up & $0.7(0.4,1.1)$ & $0.17 / 28$ & 0.24 \\
Median number of previous glaucoma surgeries & $0(0,1)$ & $6: 22$ & $0.88^{\mathrm{c}}$ \\
Median number of eyes with encircling band or belt buckle & $2 / 17$ & $16(13.8,18.5)$ \\
Site of implantation Inferior: Superior & $2: 15$ & $2(0,2)$ \\
Median postoperative IOP & $15(10,16)$ & $2(1,2)$ & $0.001^{\mathrm{c}}$ \\
Median number of postoperative AGM & & $0.44^{\mathrm{d}}$ \\
\hline
\end{tabular}

Values are presented as mean \pm SD or Median (Interquartile range)

$B C V A$ best-corrected visual acuity, $\log M A R$ logarithm of minimum angle of resolution, $A G M$ antiglaucoma medication, IOP intraocular pressure, ST superotemporal, IT inferotemporal

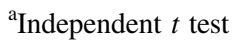

${ }^{\mathrm{b}} \mathrm{Chi}$-square test

${ }^{\mathrm{c}}$ Ranksum test

${ }^{\mathrm{d}}$ Fisher's exact test 
a

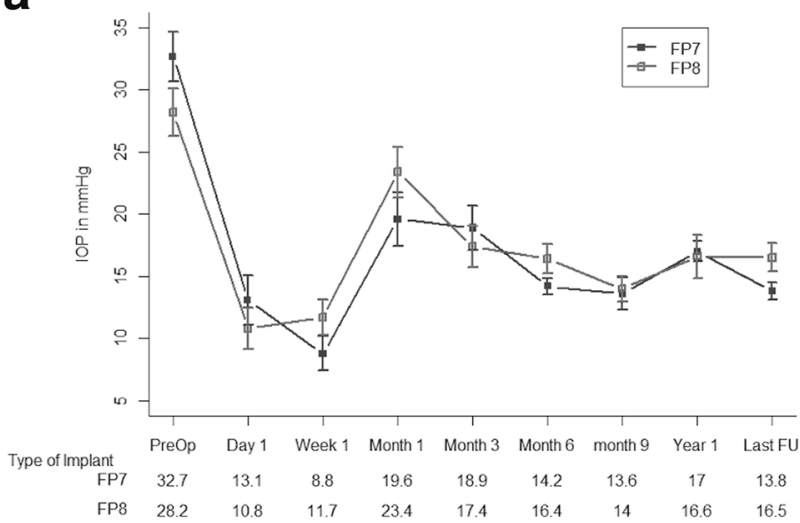

C

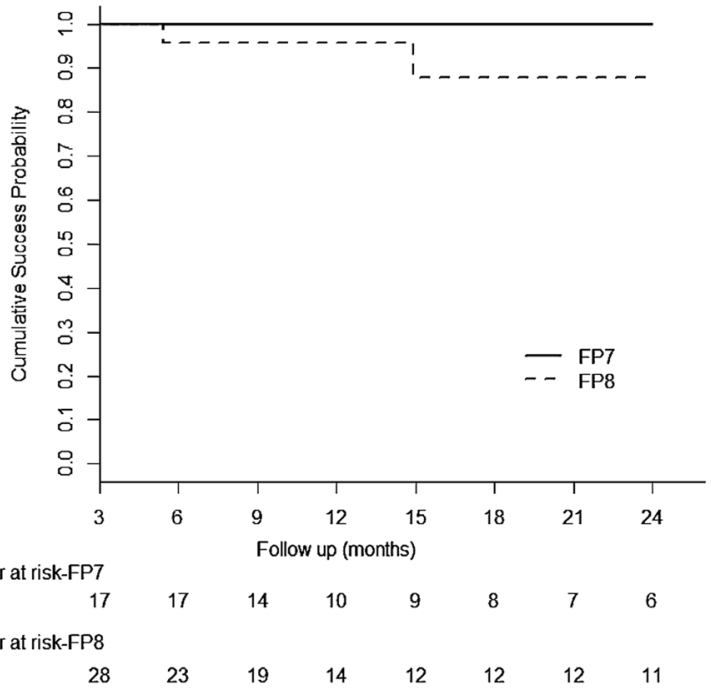

Fig. 1 a Graph showing preoperative IOP in both the groups and IOP at various time points postoperatively in the FP7 and FP8 groups. b Arrow plot showing comparison of preoperative and postoperative

The median follow-up in the FP7 group was 14.7 (9.1, 25.3) months, which was not significantly different $(p=$ $0.62)$ from the FP8 group, $9.8(6.4,34.7)$ months. Median IOP at the final follow up was $15(10,16) \mathrm{mmHg}$ and 16 $(13.8,18.5) \mathrm{mmHg}$ in the FP7 and FP8 groups, respectively, $(p=0.15)$. The preoperative and postoperative IOP at various postoperative time points indicates similar control in both groups (Fig. 1a). The median number of AGM at the final follow up were comparable between the FP8 and FP7 groups, $2(0,2)$ vs. $2(1,2),(p=0.99)$. The arrow plot (Fig. 1b) shows reduction in number of AGMs in postoperative period compared with preoperative AGM in both the groups. The median percentage drop in IOP was 55\% $(43,70 \%)$ with the FP7 implant and $53.8 \%(25,73 \%)$ with the FP8 implant $(p=0.20)$. The cumulative survival probability was $100 \%$ in the FP7 group, which was maintained for 2 years. In the FP8 group, the success was $95 \%$ at 1 year and dropped to $87.8 \%$ at 2 years. The difference in the survival by chi-square test was not significantly different b

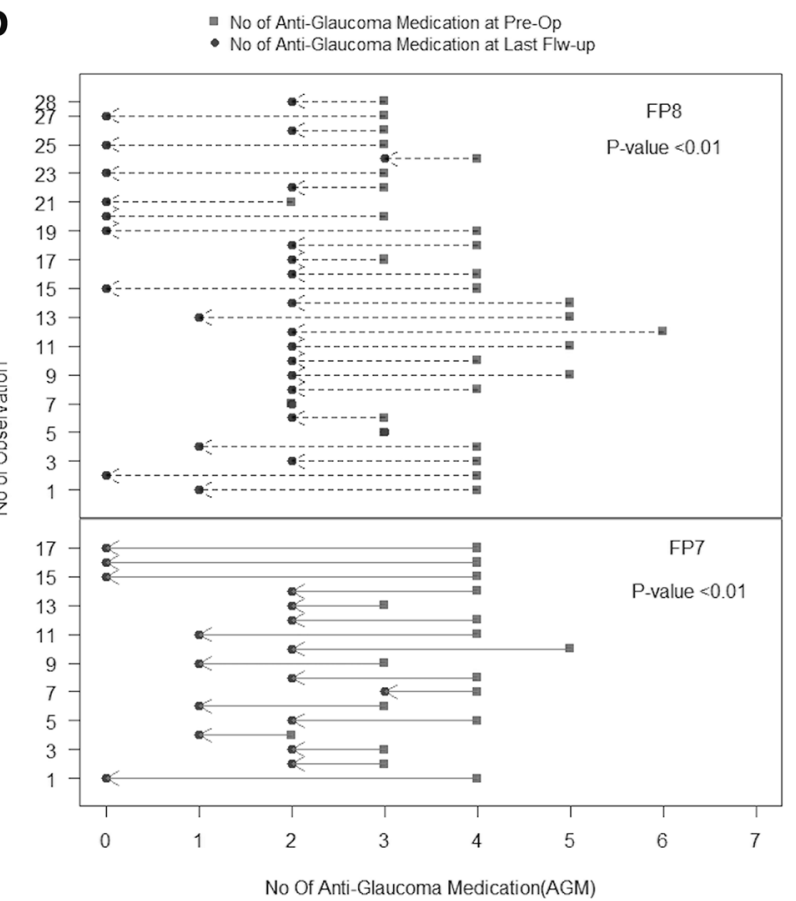

antiglaucoma medications in both the groups. c Kaplan-Meier curve showing cumulative survival probability in the FP7 and FP8 groups

$(p=0.28)$, see Fig. 1c. The median Log MAR VA at final follow up was $0.7(0.4,1.1)$ in the FP7 and $1(0.7,1.4)$ in the FP8 group $(p=0.24)$.

There were no intraoperative complications in the present study. The transient early postoperative complications that resolved spontaneously included transient hyphema (five eyes in FP7 and six eyes in FP8 group), early hypotony (one eye in FP7 group), and increased inflammation (one eye in FP8 group). Conjunctival retraction or wound dehiscence occurred in seven eyes (one eye in FP7 and six eyes in FP8 group) around first postoperative week, which was conservatively managed with oral doxycycline $100 \mathrm{mg}$ (capsule Doxt SL, Dr Reddy's Laboratories Ltd) twice daily for 2 weeks and topical lubricants. Figure 2 shows preoperative photograph of an eye with encircling band with severe conjunctival scarring in all quadrants $(\mathrm{a}-\mathrm{c})$ and postoperative photographs of the same eye following an inferonasal FP8 implantation. Note the intact conjunctival suture on day 1 (d), conjunctival gape or dehiscence at 1 week (e), 
Fig. 2 a-c The preoperative slit lamp photographs of an eye with post vitreoretinal surgery glaucoma, with encircling band and severe conjunctival scarring in all quadrants, and staphyloma in the superior and temporal quadrants. $\mathbf{d}-\mathbf{f}$ The postoperative slit lamp photographs of the same eye following inferonasal FP8 implantation. Note: the intact conjunctival suture on day 1 (d), conjunctival gape or dehiscence at 1 week (e), and complete conjunctival closure by 3 weeks (f)

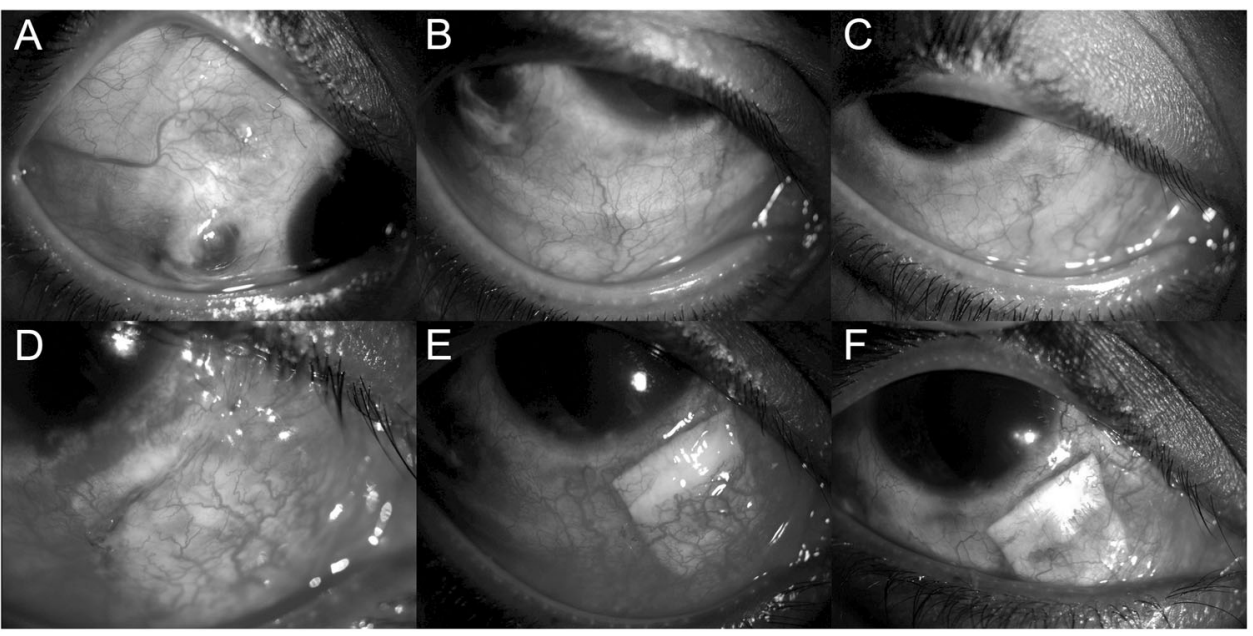

Table 2 The comparison of complications between FP7 and FP8 groups

\begin{tabular}{lllll}
\hline Complications & FP7 $(n=17)$ & FP8 $(n=28)$ & Onset (after surgery) & Treatment \\
\hline Early postoperative complications: resolved with conservative management & \\
Transient hyphema & 5 & 4 & 1 day & Conservative management \\
Hypotony & 1 & 0 & 1 day & Conservative management \\
Increased inflammation & 0 & 1 & 1 day & Conservative management \\
Conjunctival dehiscence & 1 & 5 & 1 week & Oral doxycycline \\
Total & $7 / 17(41 \%)$ & $10 / 28(35.7 \%)$ & $p=0.72$ & \\
Complications needing intervention & & 10 days & Wound resuturing \\
Conjunctival dehiscence & 0 & 1 & 9,10 months & Surgical repair with scleral patch graft \\
Tube erosion & 0 & 2 & & and conjunctival advancement \\
Total & $0 / 17(0 \%)$ & $3 / 28(10.7 \%)$ & $p=0.17$ & \\
\hline
\end{tabular}

which healed completely at 3 weeks (f). Oral doxycycline and precautions to prevent mechanical trauma to the wound helped in resolution of this conjunctival complication. The conjunctiva healed well with conservative management in five eyes 1 month postoperatively. In one eye with nonresolving conjunctival retraction (at 3 weeks), resuturing was performed with five interrupted 10-0 nylon sutures, and the patient was continued on oral doxycycline. The conjunctiva healed well within a month after resuturing.

No one in the FP7 group had any complications that needed intervention. However, three eyes in the FP8 group needed intervention $(3 / 28=10.7 \%, p=0.17)$. Of these, one eye needed conjunctival resuturing for retraction in the early postoperative period. Two eyes had tube erosion as late postoperative complication, one noted at 9 months and another at 10 months postoperatively. These were successfully managed with surgical repair, with scleral patch graft, and conjunctival advancement. None had device exposure/ extrusion, and there were no infection or corneal decompensation in our series. The details of complications are given in Table 2. The difference in the complication rate between the two groups although not statistically significant ( $p=0.17)$ may be clinically significant.

Three eyes in the FP8 group were considered as failure due to uncontrolled IOP with maximal medical therapy. Limited (15 shots in 1-2 quadrants) transscleral cyclophotocoagulation was performed for IOP control in two of these eyes, details of which are summarized in Table 3. There were no failures because of sight threatening complications or hypotony in either of the groups. One eye developed recurrent retinal detachment one year after AGV (in FP8 group), and the vision was restored by repeat VR surgery. IOP remained under control even after repeat retinal intervention. In this eye the VA improved after retinal detachment surgery compared with VA before the implant placement. A risk factor analysis showed no association with preoperative risk factors such as type of retinal pathology or type of VR surgery, number of previous intraocular/glaucoma surgeries, or presence of belt buckle with failure. 
Table 3 The clinical features and details of three eyes with failure in our cohort

\begin{tabular}{|c|c|c|c|c|c|c|}
\hline Serial no & Group & $\begin{array}{l}\text { IOP at failure } \\
(\mathrm{mmHg})\end{array}$ & $\begin{array}{l}\text { Time of failure } \\
\text { (months) }\end{array}$ & Reason for failure & Intervention & $\begin{array}{l}\text { IOP at last follow } \\
\text { up (mmHg) }\end{array}$ \\
\hline 1 & FP8 & 26 & 71 months & High IOP with MMT & TSCPC & 15 \\
\hline 2 & FP8 & 40 & 15 months & High IOP with MMT & $\mathrm{TSCPC}+$ Silicon oil removal & 18 \\
\hline 3 & FP8 & 40 & 6 months & High IOP with MMT & Nil intervention, not willing & Lost to follow up \\
\hline
\end{tabular}

$I O P$ intraocular pressure, $M M T$ maximal medical therapy, $T S C P C$ transscleral cyclophotocoagulation

\section{Discussion}

AGV implantation in post-VR surgery eyes with severe conjunctival scarring and space constrains is a major challenge. To the best of our knowledge there are no studies comparing the outcomes of different sizes of AGV (the FP7 and FP8 models) in such eyes.

Our study compares the safety and efficacy of the FP8 AGV with the standard FP7 AGV in adult post-VR surgery glaucoma with severe conjunctival scarring and limited space for implanting a drainage device. The median percentage drop in postoperative IOP was comparable with both the implants, regardless of the fact that the surface area of the FP8 is less than that of the FP7 implant. However, the surgical success with FP7 was slightly better than with FP8 (100\% with FP7 and $87.8 \%$ with FP8 at 2 years), but the difference was not significantly different $(p=0.28)$. In our series, none had sight threatening complications, and there was no implant exposure or extrusion, which is a major risk factor in eyes with scarred conjunctiva. Despite a smaller implant size, three eyes in the FP8 group had conjunctival and tube related complications needing intervention while none in the FP7 group had any complication. This could possibly be explained by the use of FP8 implants in eyes with scleral encircling bands and severe conjunctival scarring predisposed to these complications.

A few surgical modifications have been described in literature to facilitate implantation of glaucoma drainage devices in eyes with restricted surgical space. Latina et al. [9] and Law et al. [10] described implantation of an amputated (FP7) adult Ahmed valve. Smith et al. trimmed the wings of $250 \mathrm{~mm}^{2}$ Baerveldt implant to debulk the implant in eyes with a pre-existing episcleral encircling element [12]. The utility of paediatric implant in an adult eye is a possible option in these eyes rather than trimming a larger implant. In eyes with severe conjunctival scarring or in smaller eyes, a large sized adult implant may cause crowding, which can make conjunctival closure difficult and predispose the eye to complications such as conjunctival dehiscence, implant exposure, and extrusion [1315]. However, the outcomes of IOP control in smaller sized AGV implants in adult eyes remain a controversy. Longterm IOP control comparing non-valved implants with different surface area have conflicting results, with some studies reporting better outcomes with large sized implants, while others reporting no difference [16-20]. Hwang and Kee [21] also claimed that there was an upper limit for the increase in the surface area of the drainage device beyond which the beneficial effect on IOP is not marked. Most of these studies compared various sizes of Baerveldt devices; however, similar literature on AGV implants is scarce.

The only study in the literature comparing the different sizes of AGV (FP7 vs. FP8) in adult eyes is a retrospective study conducted by Koh et al. [11]. They did not observe significant difference in the outcomes, with a 3-year efficacy of $79.2 \%$ (5/24) for the FP8 and $72.7 \%$ (14/66) for the FP7. The study however excluded all the eyes with previous surgeries particularly eyes with post-VR surgery glaucoma, eyes with belt buckle, and those with severe conjunctival scarring.

Gupta et al. [8] reported on the long-term outcomes of FP7 implantation in eyes with post-VR surgery glaucoma (27 eyes of 27 patients), with $62 \%$ success probability at 12 months and $37 \%$ success probability at 5 years (success defined as IOP $\leq 21$ with or without AGM and needling). The success rates were significantly less compared with our series. They also reported a high complication rate of $48 \%$ (13/27 eyes) with the FP7 implants, of which $22 \%$ were sight threatening. The average follow up in their series was $17.1 \pm 18.36$ months, slightly longer than our FP7 group (median follow up, 14.7 (9.1, 25.3) months). The various vision threatening complications included tube/implant exposure causing endophthalmitis in two eyes (7\%), bullous keratopathy in two eyes (7\%), and retinal detachment in two eyes $(7 \%)$ with loss of light perception. None of the eyes in our series had sight threatening complications; one eye had recurrence of retinal detachment in the late postoperative period, which was successfully managed surgically with good visual outcome and IOP was maintained.

Al-Jazzaf et al. reported on the outcomes of S2 model AGV implant in post-VR surgery glaucoma after silicone oil injection in 11 eyes [22]. The cumulative success probability was $86 \%$ at 6 months, $76 \%$ at 1 year, and $64 \%$ at 2 years. The minor postoperative complications included hypertensive phase in $73 \%$ (eight eyes), low-grade inflammation in $45 \%$ (five eyes), choroidal effusion in $9 \%$ (one 
eye), hypotony in $9 \%$ (one eye), and hyphema in $9 \%$ (one eye). The success rates were similar to our series, and complications were also similar to the transient complications in our series. All eyes in their series had silicone oil related glaucoma post-VR surgery. However, there was no mention about the number of eyes with scleral buckle or scarred conjunctiva that needed implants. The fewer implant related complications could be attributed to non-scarred conjunctiva in the eyes that needed surgery.

The low complication rate seen in our study could be attributed to selection of smaller implant size that possibly helped to prevent early postoperative complications. The results of the FP8 group are encouraging despite severe conjunctival scarring and belt buckle in $>60 \%$ of these eyes. Early conjunctival retraction, an expected complication in eyes with severely scarred conjunctiva, was more common in the FP8 group. Conjunctival retraction can result in serious tube or plate related problems, with exposure or extrusion if it is detected late or inappropriately treated. However, early recognition and appropriate conservative management, including oral doxycycline and lubricants, helped in resolving this complication in a majority of the eyes in our series, except in one eye that needed conjunctival resuturing. Oral doxycycline, a semisynthetic tetracycline, is known to exhibit anti-inflammatory and anticollagenolytic properties by inhibition of metalloproteinase9 in sub-microbial dosages. Over expression of MMPs will result in excessive extracellular matrix degradation, leading to tissue destruction. Collagenases and other metalloproteinases require cations (calcium, zinc, and magnesium) to maintain their activity, so chelation of these ions could result in the reduced action of these enzymes. Therefore, oral doxycycline helps to promote wound healing, decrease scarring and tissue contraction/ retraction in the early postoperative period by chelating the cations [23, 24].

In our study, tube erosions were seen in two of the 28 eyes in the FP8 group, in the late postoperative period. Both the eyes had less than $0.5 \mathrm{~mm}$ of conjunctival erosion. The speculated causes of conjunctival erosion in these eyes could be inadequate anterior dissection of scarred conjunctiva, possible shorter overlap of patch graft anterior to tube entry, resorption or retraction of scleral patch graft, and mechanical trauma from lid movement and/or eye rubbing. This complication could be prevented by meticulous conjunctival dissection, adequate tube coverage with patch graft, posterior tube entry, or a pars plana tube entry. Meticulous postoperative follow up, early recognition, and appropriate management can help to manage this potentially serious complication. The erosion was appropriately treated with epithelial debridement, scleral path graft, and conjunctival advancement in both cases. The erosion was completely resolved in 1 month and no recurrence of this complication was noted until final follow-up.
In the current study, postoperatively, the VA improved by a median of one line in the FP7 group and decreased by a median of two lines in the FP8 group. The decrease in VA was related to worsening of primary pathology and none of the eyes that failed in the FP8 group had worsening of VA related to the implant. The eye that had recurrent RD also improved from preoperative Log MAR VA of 1.3 to 1.0.

The limitations of our study are its retrospective nature, small sample size, limited follow-up of 2 years, and loss to follow up. The choice of implant was at the surgeon's discretion based on the conjunctival mobility, scarring, and presence of encircling band. Hence there could be a bias in choosing the smaller FP8 implant for these eyes. However, lesser complications and similar IOP reduction with the smaller implants would validate this choice. If the study is performed over a decade, differences in surgical technique would surely influence the outcomes, especially the complications. However, only two surgeons were involved in treating the patients in this cohort, and both are trained glaucoma specialists from the same institute and they follow similar surgical technique. One surgical modification after 2011, during the later part of the study, was use of fibrin glue for the scleral patch graft with or without 10-0 nylon sutures. This variation in the surgical step did not contribute to any difference in the IOP or complications.

This study evaluated the safety and efficacy of the paediatric FP8 implant in managing post-VR surgery glaucoma and the results were comparable to the FP7 implant. However, as there were more eyes that needed additional procedure for IOP control with the FP8 implant, a longer follow up with more eyes would be needed for validating this observation. Serious complications like implant exposure and other sight threatening complications were not seen in this series. Hence, the FP8 AGV implant appears to be a viable option for the management of refractory glaucoma in adult eyes with limited conjunctival space and extensive scarring, as in post-VR surgery glaucoma. However, a prospective randomized clinical trial with similar indications and baseline characteristics, and with a longer follow up would be necessary to validate the results of the study.

\section{Summary}

\section{What was known before}

- Surgical management of post-VR surgery glaucoma is challenging due to severely scarred conjunctiva and the lack of space for the implant placement due to the preexisting encircling band or buckle.

- Amputation of FP7 implants or trimming of the Baerveldt implants were carried out to debulk the plate prior to implanting them in these eyes. 
- Published study using FP7 implantation in eyes with post-VR surgery glaucoma showed moderate success for IOP control, with high complication rate (48\%) and a close to $25 \%$ of them had sight threatening complications.

\section{What this study adds}

- In our series, by opting for a smaller FP8 implant in eyes with glaucoma with severely scarred conjunctiva (postVR surgery), we found visual outcomes and IOP control with smaller sized implant were comparable to eyes with adult implants over a 2 year follow-up.

- There were no sight threatening complications and complications that occurred in $10 \%$ of the eyes could be managed with surgical intervention.

Funding Hyderabad Eye Research Foundation.

\section{Compliance with ethical standards}

Conflict of interest The authors declare that they have no conflict of interest.

Publisher's note Springer Nature remains neutral with regard to jurisdictional claims in published maps and institutional affiliations.

\section{References}

1. Gedde SJ. Management of glaucoma after retinal detachment surgery. Curr Opin Ophthalmol. 2002;13:103-9.

2. Singh D, Chandra A, Sihota R, Kumar S, Gupta V. Long-term success of mitomycin-augmented trabeculectomy for glaucoma after vitreoretinal surgery with silicone oil insertion: a prospective case series. Retina. 2014;34:123-8.

3. Rotchford AP, Jayasawal R, Madhusudhan S, Ho S, King AJ, Vernon SA. Transscleral diode laser cycloablation in patients with good vision. Br J Ophthalmol. 2010;94:1180-3.

4. Sivagnanavel V, Ortiz-Hurtado A, Williamson TH. Diode laser trans-scleral cyclophotocoagulation in the management of glaucoma in patients with long-term intravitreal silicone oil. Eye. 2005; 19:253-7.

5. Ghazi-Nouri SM, Vakalis AN, Bloom PA, Bunce C, Charteris DG. Long-term results of the management of silicone oil-induced raised intraocular pressure by diode laser cycloablation. Eye. 2005;19:765-9.

6. Scott IU, Gedde SJ, Budenz DL, Greenfield DS, Flynn HW Jr, Feuer WJ, et al. Baerveldt drainage implants in eyes with a preexisting scleral buckle. Arch Ophthalmol. 2000;118:1509-13.
7. Choudhari NS, George R, Shantha B, Neog A, Tripathi S, Srinivasan $\mathrm{B}$, et al. Ahmed glaucoma valve in eyes with preexisting episcleral encircling element. Indian J Ophthalmol. 2014;62:570-4.

8. Gupta S, Chaurasia AK, Chawla R, Kapoor KS, Mahalingam K, Swamy DR, et al. Long-term outcomes of glaucoma drainage devices for glaucoma post-vitreoretinal surgery with silicone oil insertion: a prospective evaluation. Graefe's Arch Clin Exp Ophthalmol. 2016;254:2449-54.

9. Latina MA, Gulati V. A modification of the Ahmed valve for tight places. Ophthalmic Surg, Lasers Imag. 2003;34:396-7.

10. Law RW, Li RT, Lai JS, Rao SK, Lam DS. Modification of an adult-sized Ahmed glaucoma valve for a small globe. Jpn J Ophthalmol. 2006;50:67-8.

11. Koh KM, Hwang YH, Jung JJ, Sohn YH, Kim HK. Comparison of the outcome of silicone Ahmed glaucoma valve implantation with a surface area between 96 and $184 \mathrm{~mm}(2)$ in adult eyes. Korean J Ophthalmol. 2013;27:361-7.

12. Smith MF, Doyle JW, Fanous MM. Modified aqueous drainage implants in the treatment of complicated glaucomas in eyes with pre-existing episcleral bands1. Ophthalmology. 1998;105:2237-42.

13. Smith SL, Starita RJ, Fellman RL, Lynn JR. Early clinical experience with the Baerveldt $350-\mathrm{mm} 2$ glaucoma implant and associated extraocular muscle imbalance. Ophthalmology. 1993;100:914-8.

14. Schwartz KS, Lee RK, Gedde SJ. Glaucoma drainage implants: a critical comparison of types. Curr Opin Ophthalmol. 2006;17: $181-9$.

15. Minckler DS, Vedula SS, Li TJ, Mathew MC, Ayyala RS, Francis BA. Aqueous shunts for glaucoma. Cochrane Database Syst. Rev. 2006:CD004918.

16. Lloyd MA, Baerveldt G, Fellenbaum PS, Sidoti PA, Minckler DS, Martone JF, et al. Intermediate-term results of a randomized clinical trial of the 350 - versus the 500-mm2 Baerveldt implant. Ophthalmology. 1994;101:1456-63. Discussion 63-4.

17. Roy S, Ravinet E, Mermoud A. Baerveldt implant in refractory glaucoma: long-term results and factors influencing outcome. Int Ophthalmol. 2001;24:93-100.

18. Seah SK, Gazzard G, Aung T. Intermediate-term outcome of Baerveldt glaucoma implants in Asian eyes. Ophthalmology. 2003;110:888-94

19. Sidoti PA, Dunphy TR, Baerveldt G, LaBree L, Minckler DS, Lee $\mathrm{PP}$, et al. Experience with the Baerveldt glaucoma implant in treating neovascular glaucoma. Ophthalmology. 1995;102:1107-18.

20. Siegner SW, Netland PA, Urban RC Jr, Williams AS, Richards DW, Latina MA, et al. Clinical experience with the Baerveldt glaucoma drainage implant. Ophthalmology. 1995;102:1298-307.

21. Hwang JM, Kee C. The effect of surface area expansion with pericardial membrane (preclude) in Ahmed glaucoma valve implant surgery. J Glaucoma. 2004;13:335-9.

22. Al-Jazzaf AM, Netland PA, Charles S. Incidence and management of elevated intraocular pressure after silicone oil injection. J Glaucoma. 2005;14:40-6.

23. Dubey S, Prasanth B, Acharya MC, Narula R. Conjunctival erosion after glaucoma drainage device surgery: a feasible option. Indian J Ophthalmol. 2013;61:355-7.

24. Kiranmaye T, Garudadri CS, Senthil S. Role of oral doxycycline and large diameter bandage contact lens in the management of early post-trabeculectomy bleb leak. BMJ Case Rep. 2014;2014. 